\title{
DIÁLOGOS ENTRE PEDAGOGÍA Y LITERATURA EN UN AULA UNIVERSITARIA
}

\section{DIALOGUES BETWEEN PEDAGOGY AND LITERATURE IN A UNIVERSITY CLASSROOM}

\author{
Gabriela Hernando ${ }^{1}$ \\ Yésica Soledad Arenas ${ }^{2}$
}

\begin{abstract}
Resumen:
Pensar la enseñanza de la pedagogía en diálogo con la literatura adquiere el desafío de cruzar un puente, de construirlo. Es así, que en el presente artículo nos proponemos compartir la experiencia de formación que emprendimos como parte del equipo docente de la cátedra de Pedagogía II, correspondiente a las carreras de Profesorado y Licenciatura en Ciencias de la Educación de la Universidad Nacional de La Plata, Argentina. La idea de introducir una sección literaria al menú pedagógico de las clases tuvo el sencillo propósito de incorporar otro lenguaje para conversar, dialogar y pensar sobre el contenido organizador de cada encuentro; y sobre todo, introducir otras sensibilidades en el acto de enseñar y de aprender pedagogía en las aulas universitarias. El recorrido por esta experiencia está organizado en tres secciones: en la primera, describimos brevemente el contexto curricular en el que se enmarca; luego, a modo de cartografía, trazamos los lazos entre pedagogía y literatura que intentamos tender desde nuestra intención de educadoras; y por último, en clave de cierre/aperturas reflexionamos acerca de los sentidos (posibles) de incorporar la literatura en las clases de pedagogía.
\end{abstract}

Palabras clave: Pedagogía; Literatura; Enseñanza; Diálogos.

\begin{abstract}
:
To think about teaching pedagogy in dialogue with literature poses the challenge of crossing a bridge - of building it. Thus, in this article, we aim at sharing the training experience we undertook as part of the teaching staff of the subject Pedagogy II corresponding to the Teaching and Bachelor's degree in Education course of studies of the National University of La Plata, Argentina. The idea of adding a literary section to the pedagogical menu of the lessons had the simple purpose of integrating another language to converse, dialogue and think about the structuring content of each encounter; and, above all, of introducing other sensitivities to the act of teaching and learning pedagogy in university classrooms. The journey through this experience is organized into three sections: in the first one, we briefly describe the curricular context in which it is circumscribed; then, as in cartography, we draw the links between pedagogy and literature, which we try to establish from our intention as

\footnotetext{
${ }^{1}$ Universidad Nacional de La Plata, Ensenada, Argentina. Correo electrónico: hernandomgabriela@gmail.com

${ }^{2}$ Universidad Nacional de La Plata, Ensenada, Argentina. Correo electrónico: yesicaarenas@gmail.com
} 


\section{QO DEVIR EDUCAÇÃO}

ISSN: 2526-849X

educators; and lastly, as a closing/opening statement, we reflect on the (possible) meanings of integrating literature in pedagogy classes.

Keywords: Pedagogy; Literature; Teaching; Dialogues.

«Porque un puente, aunque se tenga el deseo de tenderlo y toda obra sea un puente hacia y desde algo, no es verdaderamente puente mientras los hombres no lo crucen. Un puente es un hombre cruzando un puente.»

Julio Cortázar

\section{Introducción}

Concebir el quehacer pedagógico como la construcción de un «discurso que se ocupa de trazar puentes» (ALLIAUD y FEENEY, 2004, p. 4) entre las teorías y las acciones educativas, entre los diversos sujetos pedagógicos que habitan el campo educativo y entre los distintos saberes que permiten pensar e intervenir en educación, nos ha posibilitado construir diálogos entre nuestras clases de pedagogía y el mundo de la literatura. Es así, que en el presente artículo nos proponemos compartir la experiencia que emprendimos como parte del equipo docente de la cátedra de Pedagogía II, correspondiente a las carreras de Profesorado y Licenciatura en Ciencias de la Educación de la Universidad Nacional de La Plata, Argentina.

La idea de introducir una sección literaria al menú pedagógico de las clases no tuvo una razón grandilocuente. Simplemente nuestra intención fue incorporar otro lenguaje para conversar, dialogar y pensar sobre el contenido organizador de cada encuentro; y sobre todo, introducir otras sensibilidades en el acto de enseñar y de aprender pedagogía en las aulas universitarias.

El recorrido que les proponemos para compartir este puente entre pedagogía y literatura se organiza en tres secciones: en la primera, describiremos brevemente el contexto curricular en el que se enmarca dicha experiencia; luego, a modo de cartografía, trazaremos los lazos entre pedagogía y literatura que intentamos tender desde nuestra intención de educadoras; y por último, en clave de cierre/aperturas reflexionaremos acerca de los sentidos de incorporar la literatura en las clases de pedagogía.

Pensar la enseñanza de la pedagogía en diálogo con la literatura adquiere el desafío de cruzar el puente, de construirlo; en palabras de Jorge Larrosa y Carlos Skliar es una apertura a lo dicho y lo imposible de decir, una suerte de conversación, «...modos de leer y modos de 


\section{QO DEVIR EDUCAÇÃO}

ISSN: 2526-849X

escribir para ir dibujando caminos que no sabemos a dónde conducen, escritos y leídos en una lengua que podemos llamar nuestra...» (LARROSA y SKLIAR, 2013, p. 23).

\section{El contexto curricular de la experiencia}

La asignatura Pedagogía II forma parte del tercer año de las carreras de Profesorado y Licenciatura en Ciencias de la Educación de la Universidad Nacional de La Plata, dentro del trayecto de la formación básica. Además, en el contexto curricular de las carreras oficia como materia de cierre del eje de la formación pedagógica. En este marco, Pedagogía II se propone profundizar, al mismo tiempo que retomar, preguntas, problemas, figuras y quehaceres frecuentemente asociados a la pedagogía. Como es sabido, la diversidad de usos y acepciones del término obliga a realizar una serie de recortes y omisiones inevitables.

Los contenidos que conforman la propuesta atienden a un criterio de selección minimalista en tanto nos posibilita adquirir profundidad en su abordaje y estudio. A su vez, están organizados en una unidad de aperturas y en dos unidades temáticas, de las cuales una de ellas oficia como eje articulador de un número de preguntas y conceptos que buscan describir, sistematizar y problematizar el funcionamiento propio del campo pedagógico, caracterizado por la multiplicidad y variedad de reflexiones, enfoques y perspectivas.

En un inicio, abrimos el espacio de diálogo y reflexión en torno a una serie de nociones como el pensamiento, la crítica, la pregunta y el lenguaje con el objeto de analizar cómo leemos, escribimos y pensamos en pedagogía. En palabras de Jorge Larrosa (2013), una invitación a que los/as estudiantes puedan ponerse a sí mismo en juego con lo que dicen y piensan y de este modo disponerse, en términos de Paulo Freire (2016), a una práctica-teórica: pensar desde sí el quehacer educativo. Luego, el foco de la reflexión se sitúa alrededor de una serie de figuras de maestras/os pedagogas/os o pedagogas/os maestras/os que representan pensamientos y prácticas pedagógicas diversas, que atienden a diferentes temporalidades y contextos. Las figuras propuestas: inventor en el pensamiento de Simón Rodríguez; liberador en la praxis de Paulo Freire; ignorante en la visión de Jacques Rancière; silvestre por Estanislao Antelo; y tortillera por Valeria Flores, invitan a los/as estudiantes a pensar en distintos modos de ejercer el oficio de enseñar, de sus sentidos pedagógicos, políticos e históricos, desde los cuales condensar, reflexionar, tensionar y cuestionar el campo pedagógico. Por último, como cierre parcial del recorrido, priorizamos ahondar en las nociones clásicas de la pedagogía en un doble abordaje: por un lado, analizando las categorías

Revista Devir Educação, Lavras, vol.2, n.1, p.19-36, jan./jun., 2018. 


\section{QO DEVIR EDUCAÇÃO \\ ISSN: 2526-849X}

de educación, transmisión, formación y relación pedagógica, en tanto relación ética y política; por otro, retornando a las figuras de maestros/as para trabajar estas nociones en sus obras, intentando vislumbrar los sentidos y las concepciones que les otorgan las figuras mencionadas.

En este recorrido temático la introducción de la sección literaria constituyó, generalmente, el momento de cierre de cada una de las clases. La intención de que ocupe ese momento radicó en entender el cierre como una nueva apertura. Esto es, un cierre entre comillas. Un cierre diferente. Un cierre que no pretendió cerrar sentidos, sino todo lo contrario, abrirlos. Volver a la idea de apertura para continuar pensando, y pensándonos, como educadores/as en este tiempo presente. Un cierre que nos habilite a reflexionar sobre lo acontecido para continuar pensando pedagógicamente.

Los textos literarios interpelan nuestras visiones sobre el mundo y nos invitan a preguntarnos cómo viviríamos lo representado en las ficciones. Esta dimensión no es menor ya que la mayoría de las veces es la puerta de entrada para hablar de lo que los textos nos provocan (BAJOUR, 2009). En ese sentido, el lenguaje literario oficia como disparador para activar la resonancia de la clase. Es decir, que los/as estudiantes al salir de cada encuentro continúen pensando, sintiendo, preguntando sobre algunas de las cosas dichas, o no dichas, durante los encuentros. En síntesis, invitarlos a entablar diálogos entre pedagogía y literatura a partir y más allá de la clase.

La sección literaria estuvo compuesta por distintos formatos: libros álbum y cuentos ilustrados; cartas y relatos testimoniales; poesías y coplas; narraciones breves de autores extranjeros y nacionales. El criterio que ofició para la elección fue el temático, esto es vinculado al contenido a trabajar pero también para que nuestros/as estudiantes disfruten de la lectura, teniendo presente que «La elección de textos potentes, abiertos, desafiantes, que no se queden en la seducción facilista y demagógica, que provoquen preguntas, silencios, imágenes, gestos, rechazos y atracciones, es la antesala de la escucha» (BAJOUR, 2009, p. 4).

La decisión de leer en la clase y no solo pedir leer por obligación, nos permitió incorporar en el aula otras prácticas de lectura. «Podría decirse así: como es verdad que sentimos pasión por la lectura, por cierta lectura, la ofrecemos, la damos, sin más. No habría otro condimento. No lo hay» (SKLIAR, 2015, p. 81). Así, nuestros encuentros culminaban con la lectura, por parte de las docentes o de algún/a de los/as estudiantes, de los diversos textos elegidos para el desarrollo temático de esta propuesta.

Revista Devir Educação, Lavras, vol.2, n.1, p.19-36, jan./jun., 2018. 


\section{QO DEVIR EDUCAÇÃO}

ISSN: 2526-849X

\section{Trazos de una cartografía: entre pedagogía y literatura}

El armado de esta cartografía está compuesta de varios elementos: una cronología que oficia de organizador temporal, una sucesión de puntos que permiten singularizar momentos y detenerse en la travesía y el trazado de una línea que intenta tender el puente, los diálogos (posibles) entre pedagogía y literatura.

Compartimos con Gilles Deleuze algunas de sus ideas acerca del sentido de esbozar una cartografía.

«¿Qué sería una cartografía? Un lugar de donde irse, un lugar adonde ir. Lugares por donde transcurrir, o donde quedarse un tiempo. ¿Qué sería un lugar? No sólo una topología física, sino un modo de existencia en una geografía humana (una subjetivación). Un espacio para el tiempo de una historia. La cartografía indica un trayecto en extensión, que constituye un mapa. [...] Una cartografía está hecha de líneas. [...] Es necesario trazar el plano al mismo tiempo que las líneas» (SERAGOPIAN, 2009).

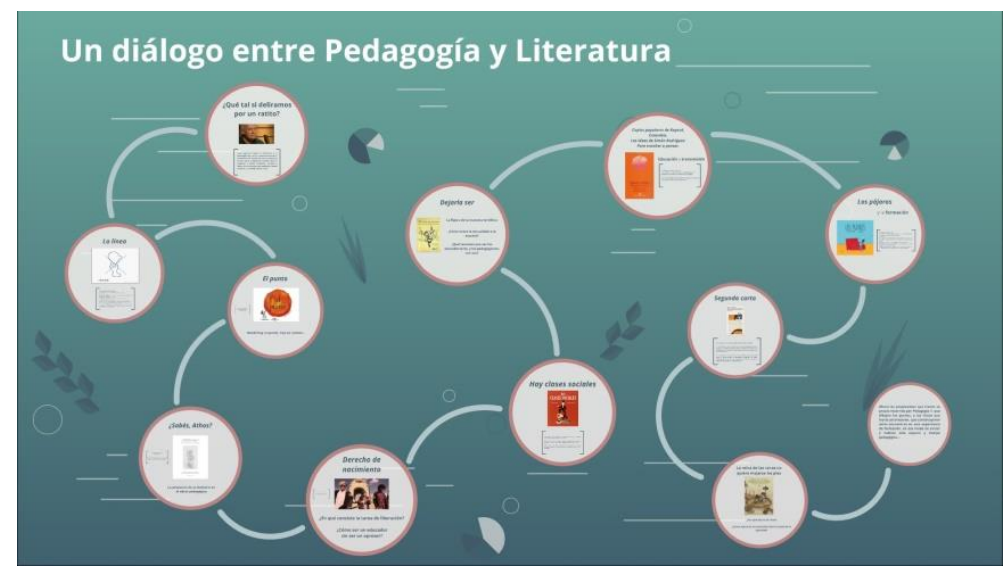

La cartografía del recorrido entre pedagogía y literatura.

Entonces, iniciamos la travesía por el punto final del recorrido puesto que ese punto es el que nos permitió des-andar el camino. Así, comenzamos el encuentro de cierre del curso de Pedagogía II con la lectura del libro álbum ${ }^{3}$ La línea de Beatriz Doumerc y Ayax Barnes

\footnotetext{
${ }^{3}$ El libro álbum es un género literario que se constituye por la relación entre el texto y las ilustraciones, donde la imagen muestra lo no dicho por la palabra, o la palabra dice lo dejado a un lado por la imagen (BAJOUR, 2009). En este tipo de libros, todos los elementos que lo constituyen (incluidos el diseño gráfico, la edición, etc.) participan en la producción del sentido. En estas obras el significado se construye de manera conjunta en el interjuego entre texto e imagen. A veces, la imagen agrega información que el texto no brinda; en ocasiones,
} 


\section{QO DEVIR EDUCAÇÃO \\ ISSN: 2526-849X}

(2010). Este libro narra las distintas acciones que un hombre puede efectuar con una línea: pasarla por arriba, pasarla por abajo... usarla y acomodarse. Pero también ese hombre con una línea puede aislarse, enredarse, dudar, retroceder y abandonarla... Además el mismo hombre con una la línea da la espalda, se incomunica, no actúa y no llega a ninguna parte... separa, divide, encierra, prohíbe, ataca y destruye. Sin embargo, ese mismo hombre tiene la posibilidad de trazar un borrón, marchar contra la historia y entonces sostener, construir, compartir... ¿Qué es entonces una línea? Los autores consideran que una línea es una sucesión de puntos y la historia es una sucesión de hechos. Los puntos hacen la línea y los hombres hacen la historia (DOUMERC y AYAX, 2010). De este modo, para nosotras la línea simboliza la pedagogía en tanto se constituyó en el trazo que nos posibilitó establecer diálogos y delinear el trayecto formativo que atravesó la propuesta.

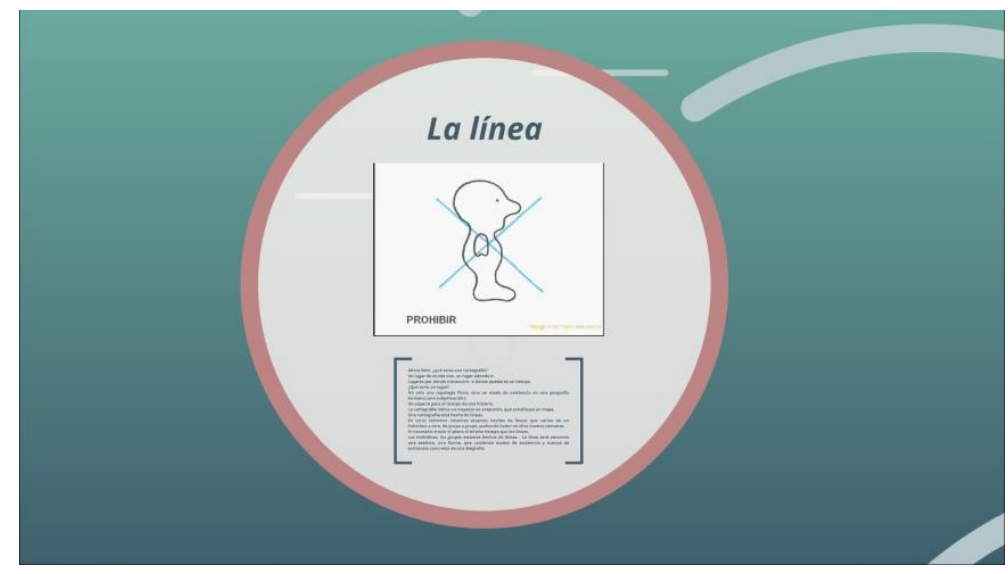

La pedagogía y la línea.

Des-andando ese trayecto nuestro punto de partida fue problematizar cómo nos relacionamos con la pedagogía y su saber a partir del acto de preguntar y de pensar problemas. En ese primer encuentro leímos un nuevo libro álbum: El punto de Peter $\mathrm{H}$. Deynolds (2005). Este libro relata la historia de Vashti, el niño que no sabe dibujar pero su profesora cree que sí. Ella sabe que todo el mundo tiene la capacidad creativa dentro de sí y que donde hay un punto hay un camino (DEYNOLDS, 2005). La historia de Vashti nos

completa un significado solo esbozado en el texto; otras veces, puede contradecir lo que el texto dice de manera explícita. En todos estos casos, el lector tiene oportunidades para reflexionar y discutir con otros sus interpretaciones, confirmarlas o reformularlas descubriendo pistas a lo largo de la obra (CASTEDO, MOLINARI, DAPINO, LANZ, PAIONE, PETRONE y TRAVERSO, 2008). 


\section{QO DEVIR EDUCAÇÃO \\ ISSN: 2526-849X}

habilitó a pensar en el punto como la capacidad que todos tenemos para preguntar y la relevancia de la pregunta en el acto de educar. Al mismo tiempo nos permitió analizar su contracara: la imposibilidad de la pregunta cuyo resultado son los silencios de los/as estudiantes. Aquí, nos detuvimos a pensar acerca de las distintas significaciones del silencio en el acto de educar como también preguntarnos: ¿de qué naturaleza es el silencio que nos constituye?

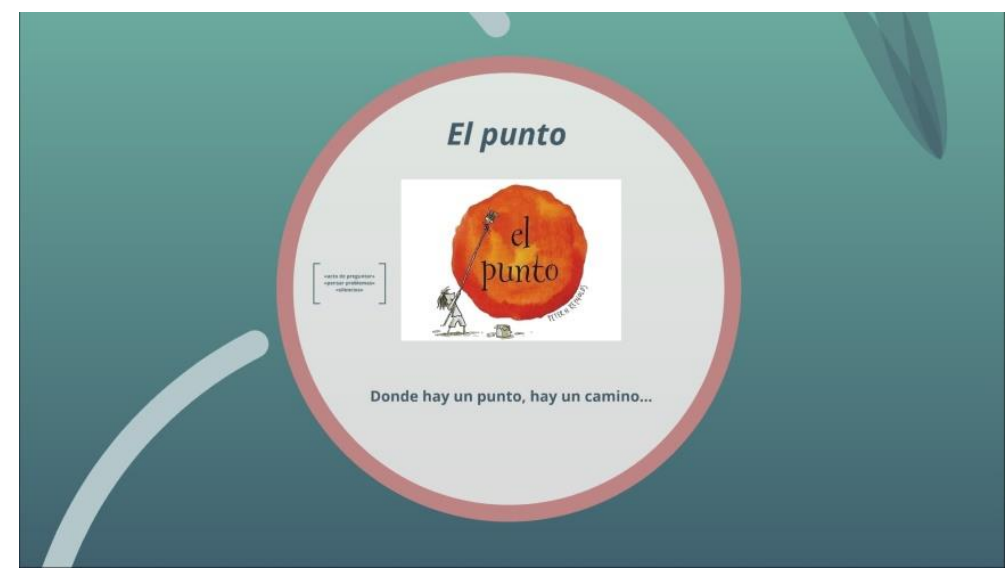

El punto y la pregunta.

Reflexionamos así sobre el silencio activo, producto de la movilización del pensamiento que hace imposible poder decir lo que se piensa (FREIRE, 2002). El silencio pasivo, como resultado de la interiorización del papel paciente que ha reservado el aula tradicional a los/as estudiantes (SHOR, 2014). El silencio indiferente, fundado en el derecho de abstenerse, en la libertad que tiene el otro de decidir si quiere o no entrar en el intercambio (ANTELO, 2005). El silencio divague, en tanto desconexión con las condiciones intolerables de una clase y como variación de la alienación producida por la pedagogía de la transferencia de conocimiento (SHOR, 2014). El silencio impuesto, provocado por prácticas autoritarias que legitimándose como un simple acto de poder establecen vínculos de dependencia y sujetos silenciados: el autor habla y decide por los demás (NASSIF, 1980). Por último, el silencio como un modo de conocer, como la ignorancia consciente de aquellos conocimientos que quedan fuera del parámetro de la normalidad y por tanto forman parte del dominio de lo impensable y de lo moralmente reprensible (FLORES, 2008).

La intervención de la maestra de Vashti: Haz solo una marca y mira adonde te lleva (DEYNOLDS, 2005) nos condujo a analizar que el pensar además de la pregunta requiere de problemas que problematicen, problemas que se sostienen en la pasión por aprender y no en 


\section{QO DEVIR EDUCAÇÃO}

ISSN: 2526-849X

la ilusión de saber (DUSCHATZKY y AGUIRRE, 2013). A su vez, el pensar conlleva un lenguaje específico, el de la experiencia. En palabras de Jorge Larrosa (2013), un lenguaje que permite hablar y escribir, leer y escuchar y pensar en nombre propio: con palabras e ideas propias. Un lenguaje en donde el yo se incluye en el nosotros pero que, además, posibilita articular textos y contextos, un pensamiento que dialoga con la realidad, con nuestra realidad.

Luego de darle apertura a la asignatura, comenzamos a trabajar con la figura del maestro liberador: Paulo Freire. Tres preguntas articularon su abordaje inicial: ¿cómo Freire llegó a ser Freire?, ¿en qué escenario político-educativo y en el debate de qué corrientes emerge su pensamiento pedagógico?, y ¿qué estilo caracteriza a la producción freireana? Allí, nos detuvimos en un nuevo punto a partir de la lectura del cuento: «¿Sabés, Athos?» de Iris Rivera y María Wernicke (2013) perteneciente al libro Quien soy: relatos sobre identidad, nietos y reencuentros.

Esta nueva historia relata el diálogo amistoso entre una niña y su perro, Athos, en su condición de hija de padres desaparecidos a partir de la dictadura militar de 1976, la «más violenta y tenebrosa de la historia argentina» (CAVAROZZI, 2002, p. 68). En uno de esos diálogos la niña expresa: «Bueno: dicen que yo no soy Bettina, que soy Candela. ¿Cómo voy a ser Candela si tengo cara de Bettina?» (RIVERA Y WERNICKE, 2013, p. 38), mostrando lo conflictivo de los procesos identitarios y de filiación producto de las crueldades de los gobiernos autoritarios.

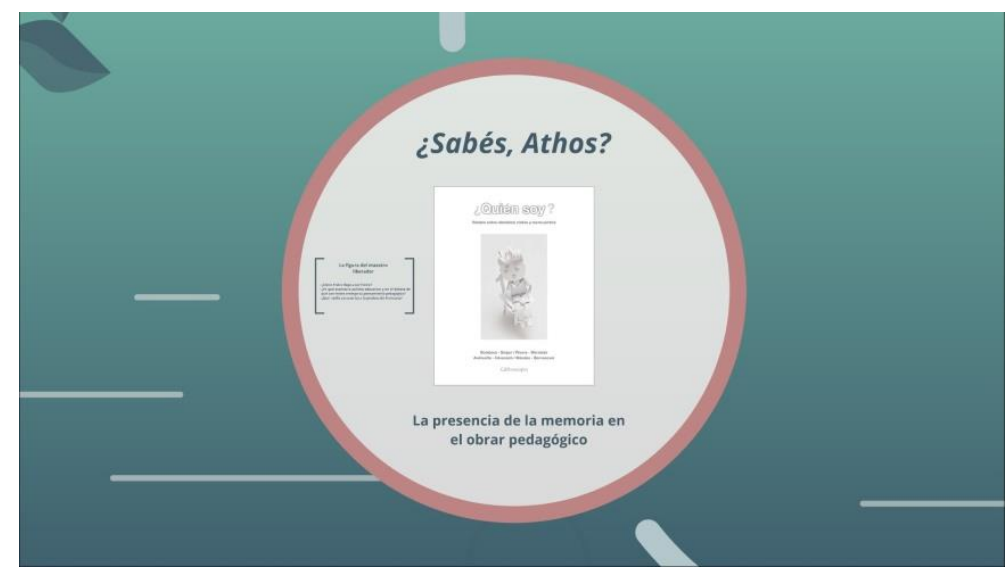

La pedagogía y la memoria.

La elección de esta historia, a diferencia de las anteriores, no la efectuamos a partir de un criterio temático sino celebrativo. Fue la forma que encontramos para conmemorar con nuestros/as estudiantes el día de la Memoria, la Verdad y la Justicia, que todos los 24 de 


\section{QO DEVIR EDUCAÇÃO \\ ISSN: 2526-849X}

marzo se recuerda en la Argentina para no olvidar los crímenes de lesa humanidad ocasionada por la última dictadura militar (1976-1983). No obstante, advertimos que la historia de vida de Bettina (o Candela) se entrelazaba con otras, en este caso con la del pedagogo brasileño Paulo Freire, objeto de estudio de nuestras clases. La cárcel, el exilio forzado, la errancia por diferentes países por causas políticas fue el derrotero seguido por el pedagogo de los oprimidos. Por esa razón, la necesidad de preservar la memoria en el obrar pedagógico es un punto central en la biografía de Paulo Freire.

Avanzando con nuestro recorrido, en un tercer encuentro compartimos la canción Derecho de Nacimiento de Natalia Lafourcade (2012), poesía hecha canción, propuesta por uno de los estudiantes. Esta canción expresaba para él, su sentir/pensar acerca de la figura del maestro liberador. Así, un fragmento de la canción nos dice:

«Voy a crear un canto para poder exigir/ Que no le quiten a los pobres lo que tanto les costó construir/ Para que el oro robado no aprecie nuestro porvenir/ $\mathrm{Y}$ a los que tienen de sobra nos les cueste tanto repartir/ Voy a elevar mi canto para hacerlos despertar/ A los que van dormidos por la vida sin querer mirar/ Yo no nací sin causa/ Yo no nací sin fe/ Mi corazón pega fuerte/ Para gritar a los que no sienten/ Y así perseguir a la felicidad» (LAFOURCADE, 2012).

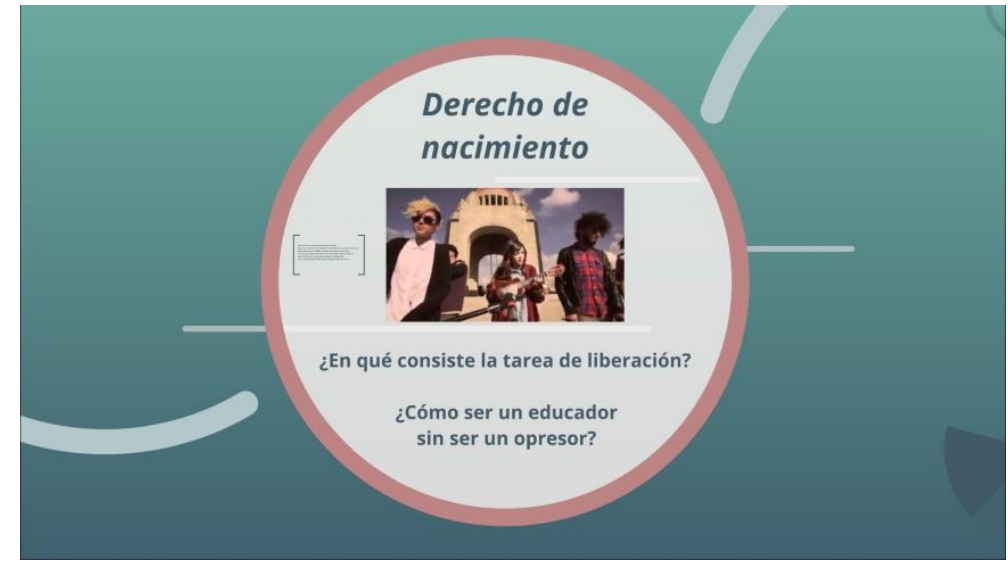

La pedagogía y las desigualdades.

La desigualdad comienza entonces a ser otro punto clave que compone esta línea que comenzamos a transitar y que permitió preguntarnos: ¿En qué consiste la tarea de liberación y cómo ser un/a educador/a sin ser un/a opresor/a?, ¿la liberación de los educandos pasa por recordarles que nacieron con causa y con fe?, ¿cuál sería nuestra causa y nuestra fe en los tiempos que nos tocan vivir?

Revista Devir Educação, Lavras, vol.2, n.1, p.19-36, jan./jun., 2018. 


\section{QO DEVIR EDUCAÇÃO \\ ISSN: 2526-849X}

Estas preguntas a su vez nos llevaron a visitar el libro álbum Hay clases sociales de Equipo Plantel y Joan Negrescolor (2015) para continuar profundizando en esta figura, en su compromiso con la liberación de los oprimidos. Su lectura introdujo la reflexión acerca de la igualdad entre los hombres a partir de las acciones de desigualdad. Desde el principio, unos hombres se aprovecharon de otros dominándolos por la fuerza. Por eso, uno son ricos y otros son pobres; unos son los dominantes, y otros, los dominados. Por eso hay clases altas que quieren conservarlo todo, clases medias que se han quedado a medias en todo y la clase trabajadora, llamada clase baja. No porque sean más bajos, ni más tontos sino porque ganan menos (EQUIPO PLANTEL y NEGRESCOLOR, 2015).

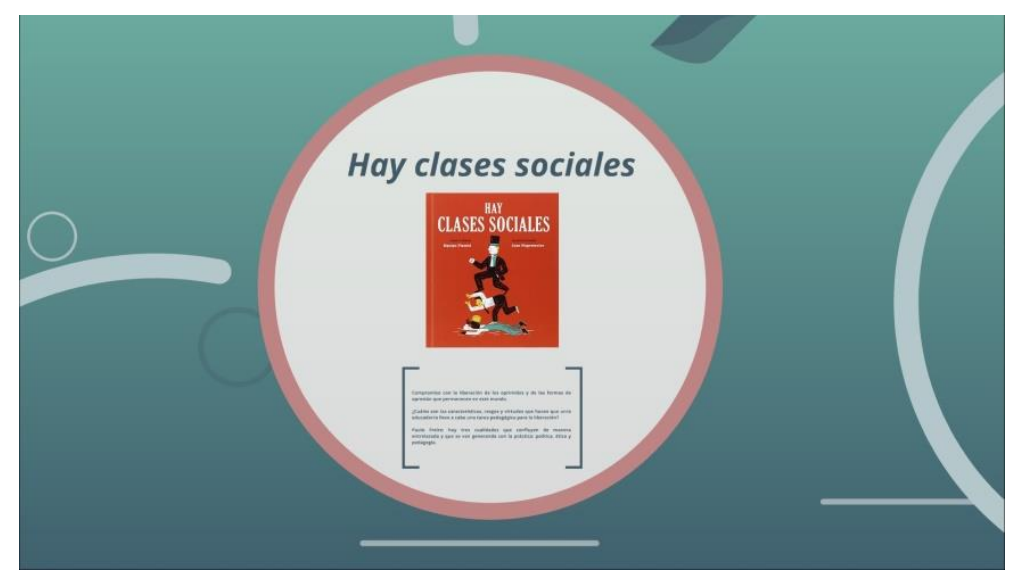

La pedagogía y las clases sociales.

Advertimos como carente de causalidad que tanto la canción de Lafourcade como el libro álbum hayan sido creados por jóvenes o pensados para niños/as puesto que nos recuerdan que las formas de opresión permanecen en este mundo. Lamentablemente las desigualdades de clase social no han desaparecido, a lo sumo se han transformado o se reactualizan en los diferentes contextos socio-históricos. Además nos recuerdan que es necesario, más bien imprescindible, que nos sigamos comprometiendo con la liberación de esas condiciones de opresión desde nuestra cotidianeidad.

El punto de la desigualdad nos llevó también a problematizar en qué consisten las características que hacen que un/a educador/a lleve a cabo una tarea pedagógica para la liberación. En el pensamiento de Paulo Freire estas cualidades se inscriben en una matriz que entrelaza tres dimensiones fundamentales e indicotomizables: la política, la pedagógica y la ética. Es más, en términos del propio Freire esas cualidades «...son predicados que se van 


\section{QO DEVIR EDUCAÇÃO \\ ISSN: 2526-849X}

generando con la práctica. Más aún son generados en la práctica en coherencia con la opción política de naturaleza crítica del educador» (FREIRE, 2002, p. 60).

Continuando con el análisis de las formas de opresión, una clase nos dedicamos a analizar otra figura de maestro/a que también trabaja este punto, la figura de la maestra tortillera. Esta nueva figura nos introdujo en la cuestión de género y de disidencia sexual y nos mostró otra de las formas de producir la desigualdad social. Interpelados por los análisis de Valeria Flores acerca de una forma diferente de ser maestra pudimos advertir la necesidad de problematizar la identidad docente y habilitar un pensar pedagógico que desobedezca la norma y las normalidades. Esas interpelaciones dieron lugar a nuevos interrogantes: ¿cómo entra la sexualidad a la escuela?, ¿qué identidades se hipervisibilizan y cuáles quedan al margen del régimen de visibilidad?, ¿qué tenemos que ver los educadores/as, y los pedagogos/as, con eso?

Ese día leímos un extracto de un relato testimonial, «Dejarla ser» del libro de Gabriela Mansilla (2014): Yo nena, yo princesa. Luana, la niña que eligió su propio nombre. Este relato elaborado en formato de diario muestra el derrotero de una mamá que supo escuchar a su hija y que emprendió una difícil lucha por el reconocimiento de la diferencia y el derecho a la identidad.

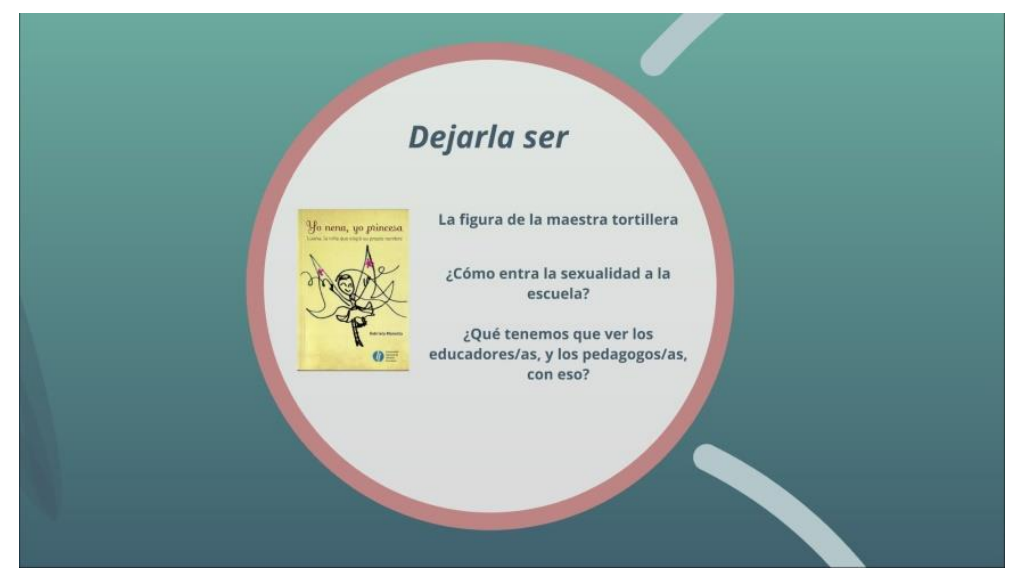

La pedagogía y el género.

De este modo, cerramos el trabajo con las figuras de maestros/as, y comenzamos a estudiar y problematizar el vocabulario propio de la pedagogía a partir de sus nociones clásicas. Un artículo de Rosa María Torres (2007) compartido por uno de los estudiantes, nos llevó a pensar cómo volver a la lectura de las figuras desde las nociones clásicas de la pedagogía indagando sobre las múltiples lecturas que hacemos, en este caso de Paulo Freire, 


\section{QO DEVIR EDUCAÇÃO \\ ISSN: 2526-849X}

pero que también funciona para las lecturas de cualquier obra, de cualquier figura y de cualquier noción: «De hecho, cuando se vuelve a leer a Freire siempre se encuentra algo nuevo. Pero para encontrar algo nuevo hay que haber avanzado uno mismo desde la última lectura» (TORRES, 2007, p. s/nro.).

Es así que en otro encuentro compartimos la lectura de dos fragmentos del escritor uruguayo Eduardo Galeano (1984), extraídos del segundo volumen de la trilogía Memoria del fuego. Las caras y las máscaras, en el cual el autor se propone narrar la historia de América Latina, revelar sus múltiples dimensiones y penetrar sus secretos. Leímos las «Coplas populares de Boyacá, Colombia» y «Las ideas de Simón Rodríguez: "Para enseñar a pensar"». La razón que inspiró esta selección radicó en problematizar las herencias (culturales, sociales, familiares, pedagógicas, etc.) porque éstas encierran una paradoja: se trata de acoger aquello que nos llega a la vez que se trata de releerlo y reinterpretarlo. En esa clase trabajamos las nociones de educación y transmisión; pensamos la educación en su relación con la sociedad mediante las funciones antagónicas de conservación/reproducción y renovación/transformación que Nassif (1980) resuelve dialécticamente; y la noción de transmisión de las herencias, éstas entendidas como la recuperación selectiva del pasado.

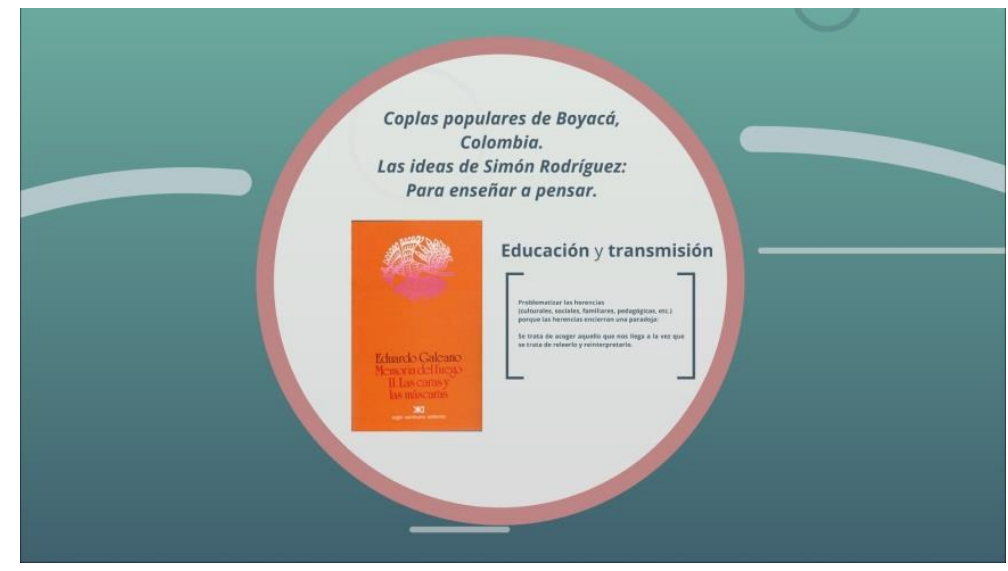

La pedagogía y las herencias.

En el encuentro siguiente incorporamos otra noción clásica de la pedagogía, la formación, que está estrechamente ligada a la noción de educación y transmisión, enunciada por Nassif en un doble sentido: en principio, afirmando que la forma de resolución de las funciones antagónicas de la educación acontecen en los sujetos y se canalizan en sus acciones; y luego, afirmando que «el verdadero punto de referencia de la educación, y, por ende, de la 


\section{QO DEVIR EDUCAÇÃO \\ ISSN: 2526-849X}

pedagogía, es el proceso de formación humana. La formación, en su acepción más vasta, es la categoría básica de la pedagogía» (NASSIF, 1980, p. 252).

Para trabajar esta temática leímos el libro álbum Los pájaros de German Zullo y Albertine (2012), que narra como un hombre conduce su camioneta hasta el borde de un precipicio para liberar un montón de pájaros coloridos. Pero, resulta que uno de ellos, un cuervo negro, no puede volar. Allí comienza una maravillosa historia entre este personaje y el pájaro negro, una historia en donde los pequeños detalles están para ser descubiertos.

La relación que se establece entre el hombre y los pájaros operó como disparador para reflexionar sobre la noción de formación como trayectoria, como dinámica del desarrollo personal a lo largo de una vida. A su vez, comprender que la formación implica hacer uso de nuestras libertades, en tanto que uno decide qué hacer con aquello que recibe, y advertir que sin trasmisión no hay formación, sin algo que ofrecer no hay algo que recibir y transformar; todos tenemos algo para dar y para recibir, que es necesario descubrir y que pasa por los pequeños detalles.

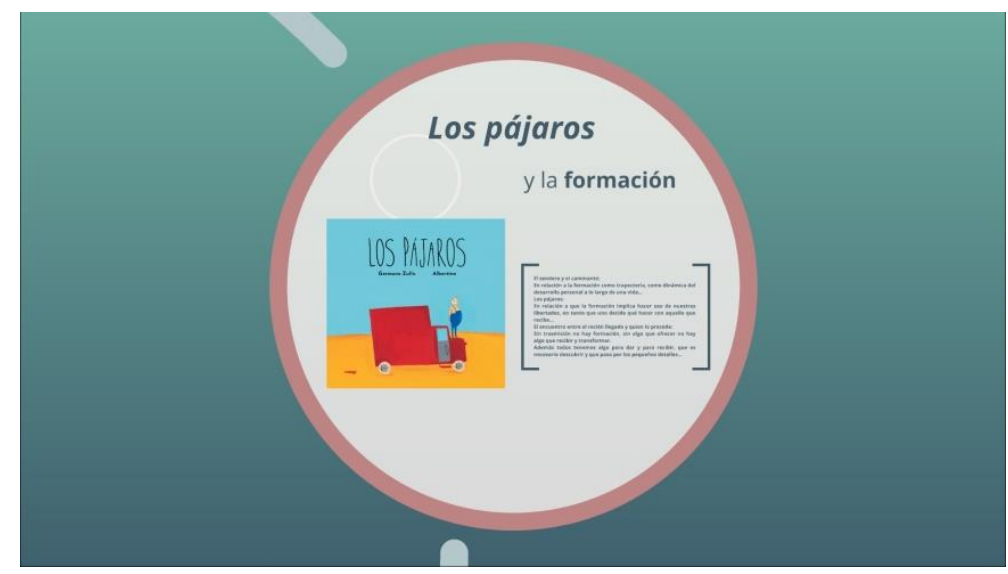

La pedagogía y la formación.

La formación, fue otro punto clave que nos permitió seguir pensando las figuras de maestros/as, es por ello que volvimos a Freire y leímos la «Segunda carta» del libro Cartas a Cristina (2008). En esta misiva el autor retorna a algunos de los acontecimientos de su infancia. Puntualmente se detiene en aquel momento en que habiendo nacido en una familia de clase media pasa a sufrir serios problemas económicos a partir de la crisis de 1929: abandonar la casa familiar para mudarse a otra más modesta, participar del mundo de los que no comían, entre otras necesidades.

Revista Devir Educação, Lavras, vol.2, n.1, p.19-36, jan./jun., 2018. 


\section{QO DEVIR EDUCAÇÃO \\ ISSN: 2526-849X}

Allí vimos como la formación en el pensamiento freireano se puede advertir en dos sentidos: la formación como trama en las que entreteje presente y pasado, y reflexiona acerca de las múltiples razones y los procesos que lo hicieron comprender cómo se convirtió en el educador que fue; y en segundo lugar, la formación como praxis, lo cual habilita a pensar cómo llegar a estar-siendo un educador liberador. Es decir, entrelazando la acción y la reflexión sobre uno mismo y sobre el mundo para la transformación.

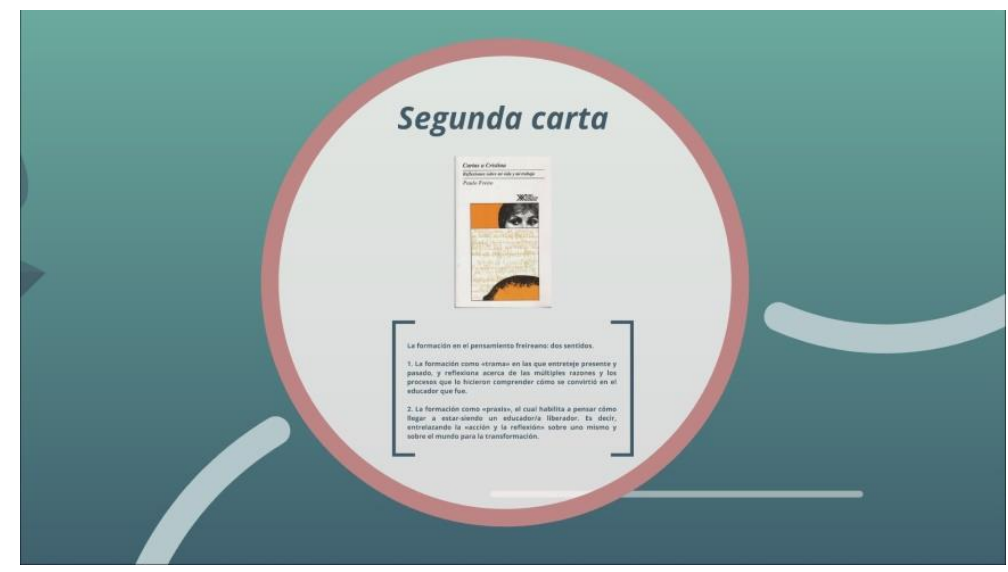

Biografía y formación.

Otra categoría, o noción, que la pedagogía se problematiza es la de autoridad. Nosotras la trabajamos desde la figura del docente y pudimos vislumbrar como al igual que las nociones de educación, transmisión y formación, la autoridad también encierra fuerzas contrarias, o se presenta como paradoja. En ese encuentro leímos La reina de las ranas no puede mojarse los pies de Davide Cali y Marcos Sòma (2013). Siguiendo el análisis que realiza Paula Pierella (2014), y entendiendo la complejidad que radica este concepto, son muchos los sentidos que se le asignan a la autoridad.

«Muchas veces se la asimila a la noción de poder, otras tantas a la de dominación y en ocasiones a la violencia. Se utiliza la palabra "autoridad" para hablar de relaciones habilitantes, centradas en el respeto y en la admiración, así como aquellas situaciones en las que imperan rasgos arbitrarios, se la asocia al temor y a la habilitación, a la autorización y al autoritarismo» (PIERELLA, 2014, p.4).

La historia de las ranas que pasaban todo el día haciendo cosas de ranas nos posibilitó problematizar los modos en que queremos ejercer la autoridad pedagógica. Un día ocurrió 


\section{QO DEVIR EDUCAÇÃO}

ISSN: 2526-849X

algo inusual en el estanque: cayó del cielo una corona. Como normalmente las coronas se ponen en la cabeza de los reyes o de las reinas, una de las ranas con la corona en la cabeza comenzó así su reinado: ella mandaba y las otras obedecían. Hasta que un buen día una rana preguntó: ¿Por qué ella es la reina? (CALI y SÒMA, 2013). De este modo, el punto de la autoridad instaló en el grupo un nuevo interrogante: ¿Cómo opera la autoridad sobre la base de la igualdad? (PIERELLA, 2014, p. 51).

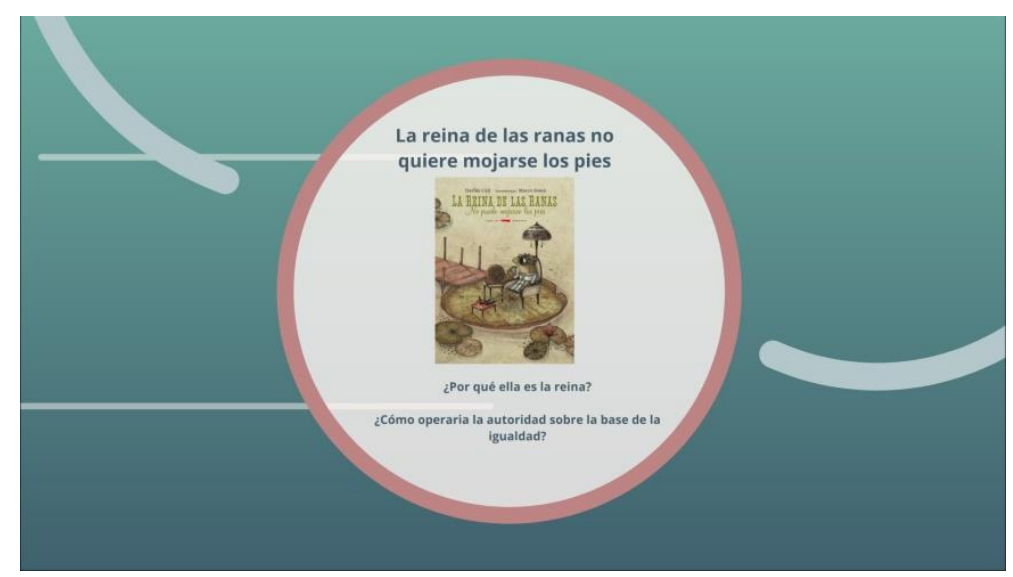

La autoridad pedagógica.

Quizás esta pregunta puede constituirse en la bisagra que articula los temas trabajados en este espacio de Pedagogía II; que articula las dos grandes unidades que componen el programa de la materia: figuras de maestros/as, y nociones clásicas de la pedagogía. Quizás, podamos pensar a esta pregunta como apertura, como un modo de relacionarnos con el saber pedagógico.

\section{A modo de cierre-apertura}

«...la literatura permite el derecho al exceso, a lo suntuoso, a salir de ese lugar atravesado por lo real. Propone un derecho a imaginar, nos abre un camino y es un modo de conocer muy intenso. Se trata de aprender a mirar el mundo con los ojos de los otros.»

María Teresa Andruetto

¿Por qué incorporar la literatura en las clases de pedagogía? Es una pregunta que nos venimos haciendo hace algún tiempo. Una pregunta que guió la construcción de estas clases 


\section{QO DEVIR EDUCAÇÃO}

ISSN: 2526-849X

de pedagogía. La intención no solo fue unir nuestras dos pasiones, la pedagogía y la literatura, sino también encontramos en esta articulación, en este cruzar, en una apertura. Una posibilidad a pensar otras formas, otros modos de intervenir pedagógicamente.

Incorporar otros formatos que irrumpen lo cotidiano de un aula universitaria es una invitación a continuar reflexionando, como dice Andruetto: Se trata de aprender a mirar el mundo con los ojos de los otros. Y entonces qué mejor que la literatura para pensar nuestras prácticas pedagógicas, para mirar el mundo, y la educación, con los ojos de los otros.

La sección literaria en las clases despertó en nuestros/as estudiantes sensaciones encontradas. Al principio, generó desconcierto y, por qué no, cierta incomodidad. Más tarde, dio lugar al disfrute y a animarse a establecer otras relaciones con el saber pedagógico, tal vez no tan autorizadas. Esperamos así que estos diálogos entre pedagogía y literatura posibiliten la construcción de sentidos transformadores, y por qué no, la construcción de otros puentes.

\section{Referencias}

ALLIAUD, Andrea; FEENEY, Silvina. Hacia la recuperación del sentido de la Pedagogía en la formación docente. En: 2do Congreso Internacional de Educación «La Formación Docente: Evaluaciones y Nuevas Prácticas en el Debate Educativo Contemporáneo». Argentina, Universidad Nacional del Litoral, 20, 21 y 22 de octubre de 2004.

ANDRUETTO, María Teresa. Dos figuras de la literatura infantil aconsejan que los chicos lean clásicos. En: Revista $\tilde{\mathbf{N}}$, Diario Clarín, 2013. Disponible en: https://www.clarin.com/infantil-y-juvenil/maria-teresa-andruetto-ana-maria-macahdo-festivalfilbita_0_rytmcAbjv7e.html Fecha de acceso: 14 de agosto de 2017.

ANTELO, Estanislao. Notas sobre la (incalculable) experiencia de educar. En: Educar: ese acto político. 1 ed. Argentina, del estante editorial, 2005, p.173-181.

BAJOUR, Cecilia. Oír entre líneas: el valor de la escucha en las prácticas de lectura. Bogotá, Asociación Colombiana de Lectura y Escritura, 2009.

CASTEDO, Mirta; MOLINARI, Claudia; DAPINO, María; LANZ, Guillermina; PAIONE, Alejandra; PETRONE, Claudia; y TRAVERSO, Viviana. La lectura en la alfabetización inicial. Situaciones didácticas en el jardín y en la escuela. La Plata, Dirección General de Cultura y Educación de la Provincia de Buenos Aires, Subsecretaría de Educación, 2008.

CAVAROZZI, Marcelo. Autoritarismo y democracia. Argentina, Eudeba, 2002.

CORTÁZAR, Julio. Libro de Manuel. Buenos Aires, Sudamericana, 1973.

DUSCHATZKY, Silvia; AGUIRRE Elina. Des-Armando Escuelas. Argentina, Paidós, 2013. 


\section{OO DEVIR EDUCAÇÃO}

ISSN: 2526-849X

FLORES, Valeria. Entre secretos y silencios. La ignorancia como política de conocimiento y práctica de (hetero) normalización. En: Revista Trabajo Social, México, n. 18, p. 14-21, feb. 2008.

FREIRE, Paulo. Pedagogía de la Esperanza. Un reencuentro con la Pedagogía del Oprimido. Argentina, Siglo XXI, 2002.

FREIRE, Paulo. Cartas a quien pretende enseñar. Buenos Aires, Siglo XXI, 2002.

FREIRE, Paulo; FAUNDEZ, Antonio. La pedagogía de hacer preguntas. En: FREIRE, Paulo y FAUNDEZ, Antonio. Por una pedagogía de la pregunta. Crítica a una educación basada en respuestas a preguntas inexistentes. 1 ed. Buenos Aires, Siglo XXI, 2014, p. 6980 .

FREIRE, Paulo. El maestro sin recetas. El desafío de enseñar en un mundo cambiante. Buenos Aires, Siglo XXI, 2016.

LARROSA, Jorge; SKLIAR, Carlos (Coords.). Entre Pedagogía y Literatura. Buenos Aires, Miño y Dávila, 2013.

NASSIF, Ricardo. Teoría de la educación. Problemática pedagógica contemporánea. Madrid, Editorial Cincel, 1980.

PIERELLA, María Paula. La autoridad en la universidad. Vínculos y experiencias entre estudiantes, profesores y saberes. Buenos Aires, Paidós, 2014.

SHOR, Ira. ¿Hay una "cultura del silencio" en los Estados Unidos? ¿Los alumnos norteamericanos, que viven en una democracia plena, necesitan de la liberación? En: FREIRE, Paulo y SHOR, Ira. Miedo y osadía. La cotidianidad del docente que se arriesga a practicar una pedagogía transformadora. 1 ed. (especial). Buenos Aires, Siglo XXI, 2014, p. 189-219.

SKLIAR, Carlos. Desobedecer el lenguaje. (Alteridad, Lectura y Escritura). Argentina, Miño y Dávila, 2015.

SERAGOPIAN, Daniel. Una Geometría humana: Las Líneas, los Planos, los Espacios. 2009. Disponible en: http://deleuzefilosofia.blogspot.com.ar/2009/01/una-geometra-humana-las-

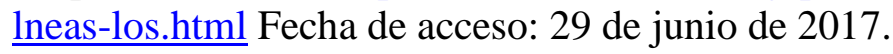

TORRES, Rosa María. Los múltiples Paulo Freire. En: Revista EAD-Educación de Adultos y Desarrollo, n. 69, 2007. Disponible en: https://www.dvv-international.de/es/educacion-deadultos-y-desarrollo/ediciones/ead-692007/el-decimo-aniversario-de-la-muerte-de-paulofreire/los-multiples-paulo-freires/ Fecha de acceso: 1 de junio de 2017.

\section{Obras literarias}


CALI, David (texto); SOMÀ, Marco (Ilustraciones). La reina de las ranas no puede mojarse los pies. Barcelona - Buenos Aires, Libros del Zorro Rojo, 2012.

DEYNOLDS, Peter H. El Punto. España, Serres, 2005.

DOUMERC, Beatriz; AYAX, Barnes. La línea. Argentina, Ediciones del Ecilpse, 2010.

EQUIPO PLANTEL (Idea y texto); NEGRESCOLOR, Joan (Ilustraciones). Hay clases sociales. Valencia, Media Vaca, 2015.

FREIRE, Paulo. Segunda Carta. En: FREIRE, Paulo. Cartas a Cristina. Reflexiones sobre mi vida y mi trabajo. 3ed. Buenos Aires, Siglo XXI, 2008, p. 38-41.

GALEANO, Eduardo. Coplas populares de Boyacá, Colombia y Las ideas de Simón Rodríguez: "Para enseñar a pensar". En: Memoria del fuego. Las caras y las máscaras. Tomo II, 4 ed. España, Siglo XXI, 1984, p. 14, 122.

MANSILLA, Gabriela. Dejarla ser. En: MANSILLA, Gabriela. Yo nena, yo princesa. Luana, la niña que eligió su propio nombre. 1 ed. Los Polvorines, Ediciones UNGS, 2016, p.65-73.

RIVERA, Iris (texto); WERNICKE, María (Ilustraciones). ¿Sabés, Athos? En: ANDRUETTO, María Teresa... [et. al.]; ilustrado por Pablo Bernasconi... [et. al.]. Quien soy: relatos sobre identidad, nietos y reencuentros. Buenos Aires, Calibroscopio, 2013.

ZULLO, German (texto); ALBERTINE (Ilustraciones). Los pájaros. Barcelona - Buenos Aires, Libros del Zorro Rojo, 2012. 